\title{
PARALLEL AFFINE IMMERSIONS WITH MAXIMAL CODIMENSION
}

\author{
LUC VRANCKEN
}

(Received January 5, 2000, revised April 23, 2001)

\begin{abstract}
We study affine immersions, as introduced by Nomizu and Pinkall, of $M^{n}$ into $\boldsymbol{R}^{n+p}$. We call $M^{n}$ linearly full if the image of $M$ is not contained in a lower dimensional affine space. Typical examples of affine immersions are the Euclidean and semi-Riemannian immersions. A classification, under an additional assumption that the rank of the second fundamental form is at least two, of the hypersurfaces with parallel second fundamental form was obtained by Nomizu and Pinkall. If we assume that the second fundamental form is parallel and $M$ is linearly full, then $p \leq n(n+1) / 2$. In this paper we completely classify the affine immersions with parallel second fundamental form in $\boldsymbol{R}^{n+n(n+1) / 2}$, obtaining amongst others the generalized Veronese immersions.
\end{abstract}

1. Introduction. We consider the standard affine space $\boldsymbol{R}^{n+p}$ equipped with its standard connection $D$. Let $M^{n}$ be a manifold equipped with a torsion free affine connection $\nabla$ and $\phi:\left(M^{n}, \nabla\right) \rightarrow\left(\boldsymbol{R}^{n+p}, D\right), p \geq 1$, be an immersion of $M^{n}$ into $\boldsymbol{R}^{n+p}$. Following [10], we call $\phi$ an affine immersion if there exists a transversal $p$-dimensional bundle $\sigma$ such that

$$
D_{X} \phi_{*}(Y)-\phi_{*}\left(\nabla_{X} Y\right) \in \sigma,
$$

for all vector fields $X$ and $Y$ which are tangent to $M^{n}$.

Another way of looking at the above equation is that each choice of transversal bundle $\sigma$ induces a particular connection on the submanifold $M$. It is immediately clear that if we equip $\boldsymbol{R}^{n+p}$ with a semi-Riemannian metric and take for $\sigma$ the associated normal bundle, then from the formula of Gauss it follows that the induced affine connection is the LeviCivita connection of the induced metric. Thus nondegenerate isometric immersions provide examples of affine immersions. Also, the equiaffine immersions, in the sense of Blaschke for hypersurfaces, and in the sense of [13], [14] or [8] for higher codimensions, provide examples of affine immersions.

For an affine immersion it is possible to introduce a bilinear form $h$, called the second fundamental form, which takes values in the transversal bundle $\sigma$ by

$$
h(X, Y)=D_{X} \phi_{*}(Y)-\phi_{*}\left(\nabla_{X} Y\right) \in \sigma .
$$

Since $\nabla$ is a torsion free affine connection, $h$ is symmetric in $X$ and $Y$. Note that both $h$ and $\nabla$ depend on the choice of transversal bundle $\sigma$. Let $\xi$ be a vector field which takes values in

2000 Mathematics Subject Classification. Primary, 53A15.

Partially supported by a research fellowship of the Alexander von Humboldt Stiftung, Germany.

Keywords and phrases. Affine differential geometry, submanifolds with parallel second fundamental form, Veronese immersions. 
$\sigma$. Similarly, as for isometric immersions, we can now introduce a normal connection $\nabla^{\perp}$ and Weingarten operators $A$ by decomposing $D_{X} \xi$ into a tangential part and a part in the direction of $\sigma$, i.e., we have the Weingarten formula which states that

$$
D_{X} \xi=-\phi_{*}\left(A_{\xi} X\right)+\nabla_{X}^{\perp} \xi
$$

Using the Weingarten formula, it is now possible to define the covariant derivative $\nabla h$ of the second fundamental form $h$ by

$$
\left(\nabla_{X} h\right)(Y, Z)=\nabla_{X}^{\perp} h(Y, Z)-h\left(\nabla_{X} Y, Z\right)-h\left(Y, \nabla_{X} Z\right) .
$$

Affine immersions for which $\nabla h$ vanishes identically are called parallel immersions. As $\nabla, h$ and $\nabla^{\perp}$ all depend on the choice of transversal bundle $\sigma$, it is easily verified that the condition that $\nabla h$ vanishes depends on the particular choice of $\sigma$ too. In Euclidean geometry, these immersions as well as their generalisations have been studied by many people, see amongst others [7]. A complete classification of the Euclidean parallel submanifolds was obtained by Ferus in [5]. As far as we know, it is still an open problem to classify the parallel semiEuclidean submanifolds.

In this paper, we will focus on parallel affine immersions. We will also assume that the immersion is linearly full. In Section 2 we will show for a linearly full parallel immersion that the dimension of the image of the second fundamental form is constant and that $p \leq$ $n(n+1) / 2$.

We will focus then on the two extremal cases, namely $p=1$ and $p=n(n+1) / 2$. In the case that $p=1$, and the rank of $h$ is at least 2, these were classified by Nomizu and Pinkall in [9], thus obtaining a characterisation of quadrics. In the case that the $p=1$ and the rank of $h$ is 1 , more possibilities can occur. These were described in [3].

The other extremal case which can occur is that when $p=n(n+1) / 2$. An example in this case is the Veronese surface in $\boldsymbol{R}^{5}$. The main purpose of this paper is to present a complete classification in this case too.

The paper is organized as follows. In Section 2 we present some basic formulas for parallel affine immersions, together with a class of immersions which can be seen as products of generalisations of the Veronese surface with quadratic submanifolds. In Section 3 we then show that a parallel affine immersion which is linearly full in $\boldsymbol{R}^{n+n(n+1) / 2}$ is affine congruent to one of the examples described in Section 2. In the case that $n=2$, this result was obtained in [6].

2. Basic equations and examples. Throughout this paper, $\phi:\left(M^{n}, \nabla\right) \rightarrow \boldsymbol{R}^{n+p}$ will denote a linearly full affine immersion. Whenever there is no possible confusion, we will identify $M^{n}$ with its image in $\boldsymbol{R}^{n+p}$. Introducing the normal curvature tensor $R^{\perp}$ by

$$
R^{\perp}(X, Y) \xi=\nabla_{X}^{\perp} \nabla_{Y}^{\perp} \xi-\nabla_{Y}^{\perp} \nabla_{X}^{\perp} \xi-\nabla_{[X, Y]}^{\perp} \xi,
$$


we get, exactly as in the Euclidean case, the equations of Gauss, Codazzi and Ricci, which are the integrability conditions for an affine immersion stating that

$$
\begin{aligned}
& R(X, Y) Z=A_{h(Y, Z)} X-A_{h(X, Z)} Y, \\
& \left(\nabla_{X} h\right)(Y, Z)=\left(\nabla_{Y} h\right)(X, Z), \\
& \left(\nabla_{X} A\right)_{\xi} Y=\left(\nabla_{Y} A\right)_{\xi} X, \\
& R^{\perp}(X, Y) \xi=h\left(X, A_{\xi} Y\right)-h\left(Y, A_{\xi} X\right),
\end{aligned}
$$

where $\left(\nabla_{X} A\right)_{\xi} Y=\nabla_{X} A_{\xi} Y-A_{\nabla_{X} \frac{1}{\xi}} Y-A_{\xi} \nabla_{X} Y$. In Euclidean geometry the Codazzi equations for $h$ and $A$ are equivalent. This is no longer true for affine immersions.

From now on we will also assume that $M$ is parallel and we denote by im $h=$ $\operatorname{span}\{h(X, Y) \mid X, Y$ are tangent vector fields $\}$. Then, we have the following lemmas:

LEMMA 1. Let $\phi:\left(M^{n}, \nabla\right) \rightarrow \boldsymbol{R}^{n+p}$ be a parallel affine immersion. Then $\operatorname{im} h$ is a differentiable subbundle of $\sigma$.

PROOF. Let $q_{1}$ and $q_{2}$ be two points in a coordinate neighborhood of $M$ and $\gamma$ a differentiable curve connecting $q_{1}$ and $q_{2}$. Let $X_{1}\left(q_{1}\right), \ldots, X_{n}\left(q_{1}\right)$ be a basis of the tangent space at $q_{1}$ and $\xi_{1}\left(q_{1}\right), \ldots, \xi_{p}\left(q_{1}\right)$ be a basis of $\sigma$ at $q_{1}$. Then it is well-known that we can extend $X_{1}, \ldots, X_{n}$ and $\xi_{1}, \ldots, \xi_{p}$ along $\gamma$ such that $\nabla_{\gamma^{\prime}} X_{i}=\nabla_{\gamma^{\prime}}^{\perp} \xi_{k}=0$. Then, if we write

$$
h\left(X_{i}, X_{j}\right)=\sum_{k=1}^{p} h_{i j}^{k} \xi_{k}
$$

it follows that

$$
0=\left(\nabla_{\gamma^{\prime}} h\right)\left(X_{i}, X_{j}\right)=\nabla_{\gamma^{\prime}}^{\perp} h\left(X_{i}, X_{j}\right)=\sum_{k=1}^{p}\left(d h_{i j}^{k} / d t\right) \xi_{k} .
$$

This implies that all $h_{i j}^{k}$ are constant along the curve $\gamma$. Consequently, the fibre dimension of im $h$ remains constant too.

Lemma 2. Let $\phi:\left(M^{n}, \nabla\right) \rightarrow \boldsymbol{R}^{n+p}$ be a parallel affine immersion. If $\phi$ is linearly full, then $\operatorname{im} h=\sigma$.

Proof. Let $W$ be any vector field in $T M \oplus \operatorname{im} h$. Thus there exist vector fields $X_{1}, X_{2}, X_{3}$ such that $W=X_{1}+h\left(X_{2}, X_{3}\right)$. Then it follows that

$$
\begin{aligned}
D_{Y} W & =D_{Y} X_{1}+D_{Y} h\left(X_{2}, X_{3}\right) \\
& =\nabla_{Y} X_{1}+h\left(Y, X_{1}\right)-A_{h\left(X_{2}, X_{3}\right)} Y+\nabla_{Y}^{\perp} h\left(X_{2}, X_{3}\right) \\
& =\nabla_{Y} X_{1}+h\left(Y, X_{1}\right)-A_{h\left(X_{2}, X_{3}\right)} Y+h\left(\nabla_{Y} X_{2}, X_{3}\right)+h\left(X_{2}, \nabla_{Y} X_{3}\right) \\
& \in T M \oplus \operatorname{im} h,
\end{aligned}
$$

implying that $T M \oplus \operatorname{im} h$ is a constant vector space along $M$. Taking a point $q$ of $M$ and coordinates in a neigborhood of that point, it now follows easily that $\phi(M)$ is contained in the affine space determined by $\phi(q)$ and $T M \oplus \operatorname{im} h$. 
Lemma 3. Let $\phi:\left(M^{n}, \nabla\right) \rightarrow \boldsymbol{R}^{n+p}$ be a parallel affine immersion. Then for all vector fields $X, Y, Z, W$ we have

$$
\begin{aligned}
h\left(X, A_{h(Z, W)} Y\right)-h\left(Y, A_{h(Z, W)} X\right)= & h\left(A_{h(Y, Z)} X, W\right)-h\left(A_{h(X, Z)} Y, W\right) \\
& +h\left(A_{h(Y, W)} X, Z\right)-h\left(A_{h(X, W)} Y, Z\right) .
\end{aligned}
$$

PROOF. Since $M$ is a parallel affine immersion, we have

$$
\nabla_{X}^{\perp} h(Y, Z)=h\left(\nabla_{X} Y, Z\right)+h\left(Y, \nabla_{X} Z\right),
$$

and thus also

$$
\begin{aligned}
\nabla_{X}^{\perp} \nabla_{Y}^{\perp} h(Z, W)= & h\left(\nabla_{X} \nabla_{Y} Z, W\right)+h\left(Z, \nabla_{X} \nabla_{Y} W\right) \\
& +h\left(\nabla_{Y} Z, \nabla_{X} W\right)+h\left(\nabla_{X} Z, \nabla_{Y} W\right) .
\end{aligned}
$$

Consequently, we have that

$$
R^{\perp}(X, Y) h(Z, W)=h(R(X, Y) Z, W)+h(Z, R(X, Y) W) .
$$

Applying now the Gauss equation (5) and the Ricci equation (8) completes the proof.

Submanifolds satisfying the conclusion of Lemma 3 are called semi-parallel submanifolds. Semi-parallel submanifolds of Euclidean spaces have been studied amongst others in [2] and [4]. The advantage of that condition is that it is an algebraic condition valid in every tangent space. The above lemma will also be the starting point of our classification in the next section.

To conclude this section, we present an example, which defines a parallel affine immersion.

EXAmple 1. Let $r, s$ be integers with $0 \leq s \leq r \leq n$. We denote by $I_{s, r+1-s}$ the $(r+1) \times(r+1)$-diagonal matrix with entries $\left[a_{i j}\right]$ satisfying:

$$
a_{i j}=0, \quad i \neq j ; \quad a_{i i}=-1, \quad i \leq s ; \quad a_{i i}=1, \quad s<i \leq r+1 .
$$

Let $v, w$ be vectors in $\boldsymbol{R}^{r+1}$. We set ${ }^{*} v={ }^{t} v I_{s, r+1-s}$ and define an inner product on $\boldsymbol{R}^{r+1}$ by $\langle v, w\rangle={ }^{*} v w$. Then, it is well-known, see [12], that the $r$-dimensional semi-Riemannian sphere $S_{s}^{r}(2)$ with index $s$ and radius 2 consists of the points $x$ satisfying

$$
{ }^{*} x x=4 \text {. }
$$

Moreover, this immersion is isometric and umbilical. Identifying $S_{s}^{r}(2)$ with its image in $\boldsymbol{R}^{r+1}$, we have that

$$
D_{X} Y=\hat{\nabla}_{X} Y-(1 / 4)^{*} X Y x,
$$

where $X, Y$ are tangent vector fields to $S_{s}^{r}(2)$ and $\hat{\nabla}$ is the Levi-Civita connection of the semiRiemannian sphere $S_{s}^{r}(2)$.

We now denote by $m=n-r$ and define a map from the semi-Riemannian product manifold $S_{s}^{r}(2) \times \boldsymbol{R}^{m}$ into an affine space $\boldsymbol{R}^{(r+1)^{2}} \times \boldsymbol{R}^{(r+1) m} \times \boldsymbol{R}^{m^{2}}$ by

$$
F(x, y)=\left(x^{*} x-(4 /(r+1)) I_{r+1}, x^{t} y,(1 / 2) y^{t} y\right) .
$$


Here the image of the first component is always a traceless $(r+1) \times(r+1)$-matrix $A$ satisfying

$$
I_{s, r+1-s}{ }^{t} A I_{s, r+1-s}=A,
$$

which is a linear subspace of dimension $r+r(r+1) / 2$. Similarly, we get that the last component is always a symmetric $m \times m$-matrix and therefore lies in a linear subspace of dimension $m+m(m-1) / 2$. Since the middle component belongs to $\boldsymbol{R}^{(r+1) m}$, it follows that the image of the map $F$ lies in a linear subspace of dimension $n+p$, where

$$
\begin{aligned}
p & =r(r+1) / 2+m(m-1) / 2+m(r+1) \\
& =(r+m)(r+m+1) / 2=n(n+1) / 2 .
\end{aligned}
$$

Note that for $s=0$ and $r=n$, we have the standard Veronese immersion. If $s \neq 0$ and $r=n$, we have the generalized Veronese immersions as introduced by Blomstrom in [1].

We denote by $y_{1}, \ldots, y_{m}$ the standard coordinates and by $\partial_{k}$ the standard parallel basis of $\boldsymbol{R}^{m}$. We denote by $X, Y, Z$ tangent vector fields to $S_{s}^{r}(2)$. Then, interpreting these vector fields in the standard way as vector fields on $S_{s}^{r}(2) \times \boldsymbol{R}^{m}$, we can define a torsion free affine connection $\nabla$ on $S_{s}^{r}(2) \times \boldsymbol{R}^{m}$ by

$$
\begin{gathered}
\nabla_{X} Y=\hat{\nabla}_{X} Y+(1 / 4)^{*} X Y \sum_{k=1}^{m} y_{k} \partial_{k}, \\
\nabla_{X} \partial_{k}=0, \quad \nabla_{\partial k} X=0, \quad \nabla_{\partial k} \partial l=0 .
\end{gathered}
$$

Now, in order to compute properties of the map $F$ we introduce the following notation. We denote by $F_{l}, 1 \leq l \leq m$, the $m \times 1$ matrix with entries $F_{l_{k 1}}$ satisfying $F_{l_{k 1}}=\delta_{l k}$ and define $E_{k l}=F_{k}{ }^{t} F_{l}, 1 \leq k, l \leq m$. Then, we get that

$$
F_{*}(X)=\left(X^{*} x+x^{*} X, X^{t} y, 0\right), \quad F_{*}\left(\partial_{k}\right)=\left(0, x^{t} F_{k},(1 / 2)\left(F_{k}{ }^{t} y+y^{t} F_{k}\right)\right) .
$$

Expressing that the Gauss formula (2) has to be satisfied, we deduce that

$$
\begin{aligned}
h\left(X, \partial_{k}\right)= & D_{X} F_{*}(\partial k)-F_{*}\left(\nabla_{X} \partial k\right)=\left(0, X^{t} F_{k}, 0\right) \\
h\left(\partial_{k}, X\right)= & D_{\partial_{k}} F_{*}(X)-F_{*}\left(\nabla_{\partial k} X\right)=\left(0, X^{t} F_{k}, 0\right) \\
h\left(\partial_{k}, \partial_{l}\right)= & D_{\partial_{k}} F_{*}\left(\partial_{l}\right)-F_{*}\left(\nabla_{\partial k} \partial l\right)=\left(0,0,\left(E_{k l}+E_{l k}\right) / 2\right) \\
h(X, Y)= & D_{X} F_{*}(Y)-F_{*}\left(\nabla_{X} Y\right) \\
= & \left(Y^{*} X+X^{*} Y+D_{X} Y^{*} x+x^{*}\left(D_{X} Y\right), D_{X} Y^{t} y, 0\right) \\
& -F_{*}\left(\hat{\nabla}_{X} Y\right)-(1 / 4)^{*} X Y \sum_{k=1}^{m} y_{k} F_{*}\left(\partial_{k}\right) \\
= & \left(Y^{*} X+X^{*} Y+\left(D_{X} Y-\hat{\nabla}_{X} Y\right)^{*} x+x^{*}\left(D_{X} Y-\hat{\nabla}_{X} Y\right),\left(D_{X} Y-\hat{\nabla}_{X} Y\right)^{t} y, 0\right) \\
& -(1 / 4)^{*} X Y\left(0, x^{t} y, y^{t} y\right) \\
= & \left(Y^{*} X+X^{*} Y-(1 / 2)^{*} X Y x^{*} x,-(1 / 2)^{*} X Y x^{t} y,-(1 / 4)^{*} X Y y^{t} y\right)
\end{aligned}
$$


Let $X_{1}, \ldots, X_{r}$ be a local orthormal basis of $S_{s}^{r}(2)$, i.e., we have that ${ }^{*} X_{i} X_{j}=\varepsilon_{i} \delta_{i j}$, where $\varepsilon_{1}=\cdots=\varepsilon_{s}=-1$ and $\varepsilon_{s+1}=\cdots=\varepsilon_{r}=1$. It is then easy to show, using in particular the metric on the Lie algebra $s o(s, r+1-s)$, that $F_{*}\left(X_{i}\right), F_{*}\left(\partial_{k}\right), h\left(X_{i}, \partial_{k}\right), h\left(\partial_{k}, \partial_{l}\right)$ and $h\left(X_{i}, X_{j}\right)$, where $i \leq j$ and $k \leq l$, are linearly independent vectors. In particular, this implies that $F$ is an immersion, that $F$ is linearly full in $\boldsymbol{R}^{n+n(n+1) / 2}$ and that the bundle spanned by $h$ is a transversal bundle. Consequently, we can consider $F$ as an affine immersion.

Since clearly

$$
D_{\partial_{k}} h\left(\partial_{l_{1}}, \partial_{l_{2}}\right)=D_{X} h\left(\partial_{l_{1}}, \partial_{l_{2}}\right)=D_{\partial_{k}} h\left(\partial_{l}, X\right)=0,
$$

it follows that

$$
\begin{gathered}
\nabla_{\partial_{k}}^{\perp} h\left(\partial_{l_{1}}, \partial_{l_{2}}\right)=\nabla_{X}^{\perp} h\left(\partial_{l_{1}}, \partial_{l_{2}}\right)=\nabla_{\partial_{k}}^{\perp} h\left(\partial_{l}, X\right)=0, \\
A_{h\left(\partial_{l_{1}}, \partial_{l_{2}}\right)} \partial_{k}=A_{h\left(\partial_{l_{1}}, \partial_{l_{2}}\right) X}=A_{h\left(\partial_{l}, X\right)} \partial_{k}=0 .
\end{gathered}
$$

The above formulas already imply that

$$
\left(\nabla_{\partial_{k}} h\right)\left(\partial_{l_{1}}, \partial_{l_{2}}\right)=\left(\nabla_{X} h\right)\left(\partial_{l_{1}}, \partial_{l_{2}}\right)=\left(\nabla_{\partial_{k}} h\right)\left(\partial_{l}, X\right)=0 .
$$

We also have that

$$
\begin{aligned}
D_{X} h\left(Y, \partial_{k}\right) & =\left(0, D_{X} Y^{t} F_{k}, 0\right)=\left(0,\left(\hat{\nabla}_{X} Y-(1 / 4)^{*} X Y x\right)^{t} F_{k}, 0\right) \\
& =h\left(\hat{\nabla}_{X} Y, \partial_{k}\right)-(1 / 4)^{*} X Y\left(0, x^{t} F_{k}, 0\right) \\
& =h\left(\nabla_{X} Y, \partial_{k}\right)-(1 / 4)^{*} X Y \sum_{l=1}^{m} y_{l} h\left(\partial_{l}, \partial_{k}\right)-(1 / 4)^{*} X Y\left(0, x^{t} F_{k}, 0\right) \\
& =h\left(\nabla_{X} Y, \partial_{k}\right)-(1 / 4)^{*} X Y\left(0, x^{t} F_{k},\left(F_{k}^{t} y+y^{t} F_{k}\right) / 2\right) \\
& =h\left(\nabla_{X} Y, \partial_{k}\right)-(1 / 4)^{*} X Y F_{*}\left(\partial_{k}\right),
\end{aligned}
$$

implying that

$$
\nabla_{X}^{\perp} h\left(Y, \partial_{k}\right)=h\left(\nabla_{X} Y, \partial_{k}\right) \quad A_{h\left(Y, \partial_{k}\right)} X=(1 / 4)^{*} X Y \partial_{k} .
$$

From the above formulas we immediately see that $\left(\nabla_{X} h\right)\left(Y, \partial_{k}\right)=0$. Therefore, by the Codazzi equation, we also have that $\left(\nabla_{\partial k} h\right)(X, Y)=0$. This can also be verified straightforwardly in the following way:

$$
\begin{aligned}
D_{\partial_{k}} h(X, Y) & =\left(0,-(1 / 2)^{*} X Y x^{t} F_{k},-(1 / 4)^{*} X Y\left(F_{k}^{t} y+y^{t} F_{k}\right)\right) \\
& =-(1 / 2)^{*} X Y F_{*}\left(\partial_{k}\right),
\end{aligned}
$$

implying that

$$
\nabla \frac{\perp}{\partial_{k}} h(Y, X)=0, \quad A_{h(Y, X)} \partial_{k}=(1 / 2)^{*} X Y \partial_{k}
$$


Finally, using that $\hat{\nabla}$ is the Levi-Civita connection of the metric, we have that

$$
\begin{aligned}
& D_{Z} h(X, Y)=D_{Z}\left(Y^{*} X+X^{*} Y-(1 / 2)^{*} X Y x^{*} x,-(1 / 2)^{*} X Y x^{t} y,-(1 / 4)^{*} X Y y^{t} y\right) \\
& =D_{Z}\left(Y^{*} X+X^{*} Y, 0,0\right)-(1 / 2)\left({ }^{*}\left(\hat{\nabla}_{Z} X\right) Y+{ }^{*} X \hat{\nabla}_{Z} Y\right)\left(x^{*} x, x^{t} y,(1 / 2) y^{t} y\right) \\
& -(1 / 2)^{*} X Y\left(Z^{*} x+x^{*} Z, Z^{t} y, 0\right) \\
& =\left(D_{Z} Y^{*} X+Y^{*} D_{Z} X+D_{Z} X^{*} Y+X^{*} D_{Z} Y, 0,0\right) \\
& \left.-(1 / 2){ }^{*}\left(\hat{\nabla}_{Z} X\right) Y+{ }^{*} X \hat{\nabla}_{Z} Y\right)\left(x^{*} x, x^{t} y,(1 / 2) y^{t} y\right) \\
& -(1 / 2)^{*} X Y\left(Z^{*} x+x^{*} Z, Z^{t} y, 0\right) \\
& =\left(\hat{\nabla}_{Z} Y^{*} X+Y^{*} \hat{\nabla}_{Z} X+\hat{\nabla}_{Z} X^{*} Y+X^{*} \hat{\nabla}_{Z} Y, 0,0\right) \\
& -(1 / 4)^{*} Z Y\left(x^{*} X+X^{*} x, 0,0\right)-(1 / 4)^{*} Z X\left(Y^{*} x+x^{*} Y, 0,0\right) \\
& -(1 / 2)\left({ }^{*}\left(\hat{\nabla}_{Z} X\right) Y+{ }^{*} X \hat{\nabla}_{Z} Y\right)\left(x^{*} x, x^{t} y,(1 / 2) y^{t} y\right) \\
& -(1 / 2)^{*} X Y\left(Z^{*} x+x^{*} Z, Z^{t} y, 0\right) \\
& =h\left(\hat{\nabla}_{Z} X, Y\right)+h\left(X, \hat{\nabla}_{Z} Y\right)-(1 / 2)^{*} X Y F_{*}(Z) \\
& -(1 / 4)^{*} Z Y\left(x^{*} X+X^{*} x, 0,0\right)-(1 / 4)^{*} Z X\left(Y^{*} x+x^{*} Y, 0,0\right) \\
& =h\left(\nabla_{Z} X, Y\right)+h\left(X, \nabla_{Z} Y\right)-(1 / 4)^{*} Z X \sum_{k=1}^{m} y_{k} h\left(Y, \partial_{k}\right) \\
& -(1 / 4)^{*} Z Y \sum_{k=1}^{m} y_{k} h\left(X, \partial_{k}\right)-(1 / 2)^{*} X Y F_{*}(Z) \\
& -(1 / 4)^{*} Z Y\left(x^{*} X+X^{*} x, 0,0\right)-(1 / 4)^{*} Z X\left(Y^{*} x+x^{*} Y, 0,0\right) \\
& =h\left(\nabla_{Z} X, Y\right)+h\left(X, \nabla_{Z} Y\right)-(1 / 4)^{*} Z X \sum_{k=1}^{m} y_{k}\left(0, Y^{t} F_{k}, 0\right) \\
& -(1 / 4)^{*} Z Y \sum_{k=1}^{m} y_{k}\left(0, X^{t} F_{k}, 0\right)-(1 / 2)^{*} X Y F_{*}(Z) \\
& -(1 / 4)^{*} Z Y\left(x^{*} X+X^{*} x, 0,0\right)-(1 / 4)^{*} Z X\left(Y^{*} x+x^{*} Y, 0,0\right) \\
& =h\left(\nabla_{Z} X, Y\right)+h\left(X, \nabla_{Z} Y\right)-(1 / 4)^{*} Z Y\left(x^{*} X+X^{*} x, X^{t} y, 0\right) \\
& -(1 / 4)^{*} Z X\left(Y^{*} x+x^{*} Y, Y^{t} y, 0\right)-(1 / 2)^{*} X Y F_{*}(Z) \\
& =h\left(\nabla_{Z} X, Y\right)+h\left(X, \nabla_{Z} Y\right) \\
& -(1 / 4)^{*} Z Y F_{*}(X)-(1 / 4)^{*} Z X F_{*}(Y)-(1 / 2)^{*} X Y F_{*}(Z),
\end{aligned}
$$

from which it follows that

$$
\begin{aligned}
\nabla_{Z}^{\perp} h(X, Y) & =h\left(\nabla_{Z} X, Y\right)+h\left(X, \nabla_{Z} Y\right) \\
A_{h(X, Y)} Z & =(1 / 4)^{*} Z Y X+(1 / 4)^{*} Z X Y+(1 / 2)^{*} X Y Z .
\end{aligned}
$$

This completes the proof that $F$ is a parallel affine immersion. 
In the next section we will prove that a linearly full parallel affine immersion of $M^{n}$ into $\boldsymbol{R}^{n+n(n+1) / 2}$ has to satisfy some further properties which will be helpfull in obtaining a classification. Since the immersion described in the example is a linearly full parallel affine immersion, it has to satisfy these properties too.

3. A classification theorem. In this section we are going to show that the immersion described in the previous section is the only parallel affine immersion of an $n$-dimensional affine manifold which is linearly full in $\boldsymbol{R}^{n+n(n+1) / 2}$. In order to do so, we first introduce some notation. We denote by $X_{1}, \ldots, X_{n}$ a local basis of tangent vector fields and define $\xi_{i j}=h\left(X_{i}, X_{j}\right)$. Clearly, we have that $\xi_{i j}=\xi_{j i}$ and because $M$ is linearly full, we also have that $\xi_{i j}, i \leq j$, are linearly independent vectors belonging to $\sigma$. We denote by $(V)^{k}$ the component of a vector field $V$ in the direction of $X_{k}$. Then, we have the following lemma.

LEMMA 4. Let $\phi:\left(M^{n}, \nabla\right) \rightarrow \boldsymbol{R}^{n+n(n+1) / 2}$ be a parallel linearly full affine immersion and $\left\{X_{1}, \ldots, X_{n}\right\}$ a tangent frame defined on a neighborhood of a point $q$ of $M^{n}$. Then there exist functions $b_{i j}, 1 \leq i, j \leq n$, with $b_{j i}=b_{i j}$ such that

$$
\begin{aligned}
& A_{\xi_{i i}} X_{i}=b_{i i} X_{i}, \\
& A_{\xi_{i i}} X_{j}=(2 / 3) b_{i j} X_{i}+(1 / 2) b_{i i} X_{j}, \\
& A_{\xi_{i j}} X_{i}=b_{i j} X_{i}+(1 / 4) b_{i i} X_{j}, \\
& A_{\xi_{i j}} X_{k}=(1 / 3) b_{j k} X_{i}+(1 / 3) b_{i k} X_{j}+(2 / 3) b_{i j} X_{k},
\end{aligned}
$$

where $i, j, k$ are mutually different indices.

Proof. Let $q \in M$ and let $\left\{X_{1}, \ldots, X_{n}\right\}$ be a tangent frame defined on a neighborhood of $q$. We introduce local functions $b_{i j}$ as follows:

(i) $b_{i i}$ is the component of $A_{\xi_{i i}} X_{i}$ in the direction of $X_{i}$,

(ii) $b_{i j}$ is the component of $A_{\xi_{i j}} X_{i}$ in the direction of $X_{i}$,

where $i$ and $j$ are different indices. Putting now $X=X_{i}, Y=Z=X_{j}$ and $W=X_{k}$, where $i, j$ and $k$ are mutually different indices, into Lemma 3 and looking at the $\xi_{i i}$ component, we deduce that $A_{\xi_{j k}} X_{j}$ has no component in the direction of $X_{i}$, where $i$ is different from $j$ and $k$.

Next, we consecutively put

(i) $X=X_{j}, Y=X_{k}, Z=W=X_{i}$; the $\xi_{j j}$ component,

(ii) $X=X_{i}, Y=X_{j}, Z=W=X_{i}$; the $\xi_{j j}$ component,

(iii) $X=X_{i}, Y=X_{j}, Z=X_{k}, W=X_{l}$; the $\xi_{j j}$ component,

in order to find that

(i) $\quad A_{\xi_{i i}} X_{k}$ has no component in the direction of $X_{j}$ with $j$ different from $i$ and $k$,

(ii) $A_{\xi_{i i}} X_{i}$ has no component in the direction of $X_{j}$ with $j$ different from $i$,

(iii) $\quad A_{\xi_{k l}} X_{i}$ has no component in the direction of $X_{j}$ with $j$ different from $k, l$ and $i$. The above already implies that

$$
A_{\xi_{i i}} X_{i}=b_{i i} X_{i}
$$


Now, we take $X=X_{i}=Z=W$ and $Y=X_{j}$. Then, using the above equation, we find that

$$
-b_{i i} \xi_{i j}+3 h\left(A_{\xi_{i i}} X_{j}, X_{i}\right)-2 h\left(A_{\xi_{i j}} X_{i}, X_{i}\right)=0 .
$$

Looking at the $\xi_{i i}$ component of the above equation gives that

$$
3\left(A_{\xi_{i i}} X_{j}\right)^{i}=2\left(A_{\xi_{i j}} X_{i}\right)^{i}=2 b_{i j}
$$

Hence

$$
\left(A_{\xi_{i i}} X_{j}\right)^{i}=(2 / 3) b_{i j}
$$

Whereas, looking at the $\xi_{i j}$-component of (9) yields that

$$
3\left(A_{\xi_{i i}} X_{j}\right)^{j}-2\left(A_{\xi_{i j}} X_{i}\right)^{j}-b_{i i}=0 .
$$

Taking $X=X_{i}=Z$ and $Y=X_{j}=W$ next, we find that

$$
2 h\left(A_{\xi_{i j}} X_{j}, X_{i}\right)-2 h\left(A_{\xi_{i j}} X_{i}, X_{j}\right)=h\left(A_{\xi_{j j}} X_{i}, X_{i}\right)-h\left(A_{\xi_{i i}} X_{j}, X_{j}\right) .
$$

Looking at the $\xi_{i j}$ component of (12), using the definition of $b_{i j}$ and (10), we get that

$$
2 b_{j i}-2 b_{i j}=(2 / 3) b_{j i}-(2 / 3) b_{i j} \text {. }
$$

Hence $b_{i j}=b_{j i}$. It also follows from (12) that

$$
2\left(A_{\xi_{i j}} X_{i}\right)^{j}=\left(A_{\xi_{i i}} X_{j}\right)^{j} .
$$

Combining this with (11), we obtain that

$$
\left(A_{\xi_{i j}} X_{i}\right)^{j}=b_{i i} / 4, \quad\left(A_{\xi_{i i}} X_{j}\right)^{j}=b_{i i} / 2 .
$$

Finally, we still have to compute $\left(A_{\xi_{i j}} X_{k}\right)^{i}$ and $\left(A_{\xi_{i j}} X_{k}\right)^{k}$. Since $\xi_{i j}=\xi_{j i}$, it follows that we can introduce functions $c_{i j k}$ and $d_{i j k}$ such that

$$
A_{\xi_{i j}} X_{k}=c_{i j k} X_{i}+c_{j i k} X_{j}+d_{i j k} X_{k} .
$$

We take $X=X_{i}=W, Y=X_{j}$ and $Z=X_{k}$. Then, we obtain that

$$
2 h\left(A_{\xi_{i k}} X_{j}, X_{i}\right)-h\left(A_{\xi_{j k}} X_{i}, X_{i}\right)=b_{i k} \xi_{i j}+(1 / 3) b_{i j} \xi_{i k} .
$$

Looking at the different components of the above equation, we deduce that

$$
d_{j k i}=2 c_{i k j}, \quad 2 c_{k i j}-c_{k j i}=(1 / 3) b_{i j}, \quad 2 d_{i k j}=c_{j k i}+b_{i k} .
$$

Combining the first and the last of the above equations, it follows that

$$
c_{j k i}+b_{i k}=2 d_{i k j}=4 c_{j k i} .
$$

Hence $c_{j k i}=(1 / 3) b_{i k}$ and $d_{i k j}=(2 / 3) b_{i k}$.

We recall that the Ricci tensor Ric associated with the curvature tensor $R$ of an affine connection is defined by

$$
\operatorname{Ric}(Y, Z)=\operatorname{trace}\{X \mapsto R(X, Y) Z\} .
$$

Using Lemma 4 we compute in the following lemma the Ricci tensor in terms of the functions $b_{i j}$. 
LEMMA 5. Let $\phi:\left(M^{n}, \nabla\right) \rightarrow \boldsymbol{R}^{n+n(n+1) / 2}$ be a parallel linearly full affine immersion and $\left\{X_{1}, \ldots, X_{n}\right\}$ a tangent frame defined on a neighborhood of a point $q$ of $M^{n}$. Then, using the notation of the previous lemma, we have that

$$
\begin{aligned}
& \operatorname{Ric}\left(X_{i}, X_{j}\right)=(n-1) b_{i j} / 3, \quad i \neq j, \\
& \operatorname{Ric}\left(X_{i}, X_{i}\right)=(n-1) b_{i i} / 4 .
\end{aligned}
$$

Proof. We consider two cases. If $i \neq j$, we have that

$$
\begin{aligned}
\operatorname{Ric}\left(X_{i}, X_{j}\right) & =\sum_{k=1}^{n}\left(R\left(X_{k}, X_{i}\right) X_{j}\right)^{k} \\
& =\sum_{\substack{k=1 \\
k \neq i, j}}^{n}\left((2 / 3) b_{i j}-(1 / 3) b_{i j}\right)+\left(R\left(X_{j}, X_{i}\right) X_{j}\right)^{j} \\
& =(n-2) b_{i j} / 3+b_{i j}-(2 / 3) b_{i j}=(n-1) b_{i j} / 3 .
\end{aligned}
$$

Finally, we have that

$$
\begin{aligned}
\operatorname{Ric}\left(X_{i}, X_{i}\right) & =\sum_{k=1}^{n}\left(R\left(X_{k}, X_{i}\right) X_{i}\right)^{k}=\sum_{\substack{k=1 \\
k \neq i}}^{n}\left(R\left(X_{k}, X_{i}\right) X_{i}\right)^{k} \\
& =\sum_{\substack{k=1 \\
k \neq i}}^{n}\left(b_{i i} / 2-b_{i i} / 4\right)=(n-1) b_{i i} / 4 .
\end{aligned}
$$

Note that it follows from the previous lemmas that the Ricci tensor of $\nabla$ is symmetric. Let $q \in M$. Since, starting with three linearly independent tangent vectors $v, u, w$ at the point $q$, we can always construct a tangent basis at the point $q$, we find by combining Lemma 4 with Lemma 5 that

$$
\begin{aligned}
A_{h(v, v)} v= & (4 /(n-1)) \operatorname{Ric}(v, v) v \\
A_{h(v, v)} u= & (2 /(n-1)) \operatorname{Ric}(u, v) v+(2 /(n-1)) \operatorname{Ric}(v, v) u, \\
A_{h(u, v)} v= & (3 /(n-1)) \operatorname{Ric}(u, v) v+(1 /(n-1)) \operatorname{Ric}(v, v) u, \\
A_{h(u, v)} w= & (1 /(n-1)) \operatorname{Ric}(u, w) v+(1 /(n-1)) \operatorname{Ric}(v, w) u \\
& +(2 /(n-1)) \operatorname{Ric}(u, v) w .
\end{aligned}
$$

Taking the appropriate limits, it is clear that the above formulas remain valid for linearly dependent $u, v, w$ too. Therefore, we have shown the following lemma: 
LEMMA 6. Let $M$ be as in the previous lemmas and let $u, v, w$ be arbitrary tangent vectors. Then

$$
A_{h(u, v)} w=(1 /(n-1)) \operatorname{Ric}(u, w) v+(1 /(n-1)) \operatorname{Ric}(v, w) u+(2 /(n-1)) \operatorname{Ric}(u, v) w .
$$

Remark that in the special case that $M$ is an isometric immersion into a Euclidean space, the formula (15) implies that the immersion is isotropic in the sense of O'Neill, see [11]. In that respect, it makes sense to define an affine immersion isotropic if there exists a symmetric bilinear form $\alpha$ such that

$$
A_{h(v, v)} v=\alpha(v, v) v
$$

It is clear that every Euclidean isotropic immersion is also an affine isotropic immersion.

The previous lemma then states that every semi-parallel affine immersion $\phi:\left(M^{n}, \nabla\right) \rightarrow$ $\boldsymbol{R}^{n+n(n+1) / 2}$ is affine isotropic. It also follows easily that a codimension one nondegenerate affine immersion is affine isotropic if and only if $A_{\xi}$ is proportional to the identity, i.e., if and only if it is an affine sphere.

So far, we have only worked at the tangent space at one point. Up to this point all results would remain valid for semi-parallel immersions too, provided that $T M \oplus \operatorname{im}(h)$ was $n+n(n+1) / 2$-dimensional. In the next steps we will explicitly use the fact that $M$ is parallel together with the Codazzi equation for $A$ in order to show that the Ricci tensor is parallel and that the rank of it is constant on $M$. This is done in the following lemmas:

LEMMA 7. Let $M$ be as before. Then $\left(\nabla_{X} \operatorname{Ric}\right)(Y, Z)=0$ for all tangent vector fields $X, Y$ and $Z$.

PROOF. We take linearly independent vector fields $X$ and $Y$. Then, we have that

$$
\left(\nabla_{X} A\right)_{h(X, X)} Y=\left(\nabla_{Y} A\right)_{h(X, X)} X .
$$

Computing the left-hand side of (20), we find that

$$
\begin{aligned}
\left(\nabla_{X} A\right)_{h(X, X)} Y= & \nabla_{X} A_{h(X, X)} Y-A_{\nabla_{X}^{\perp} h(X, X)} Y-A_{h(X, X)} \nabla_{X} Y \\
= & \nabla_{X}((2 /(n-1)) \operatorname{Ric}(X, Y) X+(2 /(n-1)) \operatorname{Ric}(X, X) Y) \\
& -2 A_{h\left(\nabla_{X} X, X\right)} Y-A_{h(X, X)} \nabla_{X} Y \\
= & \nabla_{X}((2 /(n-1)) \operatorname{Ric}(X, Y) X+(2 /(n-1)) \operatorname{Ric}(X, X) Y) \\
& -2\left((1 /(n-1)) \operatorname{Ric}\left(\nabla_{X} X, Y\right) X+(1 /(n-1)) \operatorname{Ric}(X, Y) \nabla_{X} X\right. \\
& \left.+(2 /(n-1)) \operatorname{Ric}\left(\nabla_{X} X, X\right) Y\right) \\
& -\left((2 /(n-1)) \operatorname{Ric}\left(\nabla_{X} Y, X\right) X+(2 /(n-1)) \operatorname{Ric}(X, X) \nabla_{X} Y\right) \\
= & (2 /(n-1))\left(\nabla_{X} \operatorname{Ric}\right)(X, Y) X+(2 /(n-1))\left(\nabla_{X} \operatorname{Ric}\right)(X, X) Y,
\end{aligned}
$$

whereas the right-hand side of (20) gives that 


$$
\begin{aligned}
\left(\nabla_{Y} A\right)_{h(X, X)} X= & \nabla_{Y} A_{h(X, X)} X-A_{\nabla_{Y}^{\frac{1}{Y}} h(X, X)} X-A_{h(X, X)} \nabla_{Y} X \\
= & \nabla_{Y}(4 /(n-1)) \operatorname{Ric}(X, X) X-2 A_{h\left(\nabla_{Y} X, X\right)} X-A_{h(X, X)} \nabla_{Y} X \\
= & \nabla_{Y}(4 /(n-1)) \operatorname{Ric}(X, X) X-2\left((3 /(n-1)) \operatorname{Ric}\left(\nabla_{Y} X, X\right) X\right. \\
& \left.+(1 /(n-1)) \operatorname{Ric}(X, X) \nabla_{Y} X\right) \\
& -\left((2 /(n-1)) \operatorname{Ric}\left(\nabla_{Y} X, X\right) X+(2 /(n-1)) \operatorname{Ric}(X, X) \nabla_{Y} X\right) \\
= & (4 /(n-1))\left(\nabla_{Y} \operatorname{Ric}\right)(X, X) X .
\end{aligned}
$$

Comparing both sides of (20), it follows that for linearly independent $X$ and $Y$, we have that

$$
\begin{aligned}
& \left(\nabla_{X} \operatorname{Ric}\right)(X, X)=0, \\
& 2\left(\nabla_{Y} \operatorname{Ric}\right)(X, X)-\left(\nabla_{X} \operatorname{Ric}\right)(X, Y)=0 .
\end{aligned}
$$

Since (21) remains valid for all vectors $X$, it follows that

$$
\left(\nabla_{a X+b Y} \operatorname{Ric}\right)(a X+b Y, a X+b Y)=0
$$

for all values of $a$ and $b$. The above equation can be seen as a polynomial equation in $a$ and $b$ which vanishes identically. Therefore every coefficient has to vanish. Using that Ric is symmetric and computing the coefficient of $a^{2} b$, we find that

$$
\left(\nabla_{Y} \operatorname{Ric}\right)(X, X)+2\left(\nabla_{X} \operatorname{Ric}\right)(X, Y)=0 .
$$

Combining this with (22), it follows that

$$
\begin{aligned}
& \left(\nabla_{Y} \operatorname{Ric}\right)(X, X)=0, \\
& \left(\nabla_{X} \operatorname{Ric}\right)(X, Y)=0 .
\end{aligned}
$$

As (23) remains valid for all vectors $X$ and $Y$, we also have

$$
\left(\nabla_{Y} \operatorname{Ric}\right)(X+Z, X+Z)=0 .
$$

Since Ric is symmetric, this last equation implies that $\left(\nabla_{Y} \operatorname{Ric}\right)(X, Z)=0$.

Let $r_{q}$ denote the rank of the Ricci tensor at the point $q$. Then, we have:

LEMMA 8. Let $\phi:\left(M^{n}, \nabla\right) \rightarrow \boldsymbol{R}^{n+n(n+1) / 2}$ be a parallel affine immersion. Then the rank $r_{q}$ of the Ricci tensor at the point $q$ is independent of $q$.

PROOF. Let $q_{1}$ and $q_{2}$ be two points in a coordinate neighborhood of $M$ and $\gamma$ be a differentiable curve connecting $q_{1}$ and $q_{2}$. Let $X_{1}\left(q_{1}\right), \ldots, X_{n}\left(q_{1}\right)$ be a basis of the tangent space at $q_{1}$. Then it is well-known that we can extend $X_{1}, \ldots, X_{n}$ along $\gamma$ such that $\nabla_{\gamma^{\prime}} X_{i}=$ 0 . Then, it follows that

$$
0=\left(\nabla_{\gamma^{\prime}} \operatorname{Ric}\right)\left(X_{i}, X_{j}\right)=(d / d t)\left(\operatorname{Ric}\left(X_{i}, X_{j}\right)\right) .
$$

This implies that $\operatorname{Ric}\left(X_{i}, X_{j}\right)$ remains constant along the curve $\gamma$. Consequently, the rank of the Ricci tensor is constant. 
We denote the rank of the Ricci tensor by $r$. Since it is constant on $M$, we can define a distribution $T_{0}$ on $M$ by

$$
T_{0}=\{Z \mid \operatorname{Ric}(Y, Z)=0 \text { for all vector fields } Y\} .
$$

The dimension of this distribution equals $m$, where $m=n-r$. Let $Z \in T_{0}$, and let $X$ and $Y$ be arbitrary vector fields. Since $0=\left(\nabla_{X} \operatorname{Ric}\right)(Y, Z)=-\operatorname{Ric}\left(Y, \nabla_{X} Z\right)$, it follows that $\nabla_{X} Z \in T_{0}$ for $Z \in T_{0}$. This shows that the distribution $T_{0}$ is parallel with respect to $\nabla$.

The next lemmas will then show how to construct a suitable basis of the distribution $T_{0}$ and how to define a suitable complementary distribution. However, before doing this, we need the following lemma about the solution of differentiable equations on affine differentiable manifolds:

LEMMA 9. Let $m$ be a positive integer and $\alpha_{i j}$ and $\beta_{i}, i, j=1, \ldots, m$, one-forms on $M$. Then, if those one-forms satisfy

$$
\begin{aligned}
& d \beta_{i}(X, Y)-\sum_{j=1}^{m}\left(\alpha_{i j}(X) \beta_{j}(Y)-\alpha_{i j}(Y) \beta_{j}(X)\right)=0, \\
& d \alpha_{i j}(X, Y)-\sum_{j_{2}=1}^{m}\left(\alpha_{i j_{2}}(X) \alpha_{j_{2} j}(Y)-\alpha_{i j_{2}}(Y) \alpha_{j_{2} j}(X)\right)=0,
\end{aligned}
$$

then the system of partial differential equations given by:

$$
X\left(f_{i}\right)=\sum_{j=1}^{m} \alpha_{i j}(X) f_{j}+\beta_{i}(X),
$$

has a solution.

PROOF. By introducing a function $f_{m+1}=1$ and forms $\alpha_{m+1 i}=0, \alpha_{m+1 m+1}=0$ and $\alpha_{i m+1}=\beta_{i}$, we see that it is sufficient to prove the lemma in the case that all $\beta_{i}$ vanish identically. Using local coordinates $\left\{x_{1}, \ldots, x_{n}\right\}$, we see that it is then sufficent to prove that a system

$$
\partial_{i} f=A_{i} f,
$$

where $\partial_{i}=\left(\partial / \partial x_{i}\right), f={ }^{t}\left(f_{1}, \ldots, f_{m}\right)$ and $A_{i}$ is an $m \times m$-matrix, has a solution provided that

$$
\partial_{i}\left(A_{j}\right)-\partial_{j}\left(A_{i}\right)-\left[A_{i}, A_{j}\right]=0 .
$$

We now show the above claim by induction on the number of variables $n$. If $n=1$, the condition becomes trivial and the claim follows immediately from the existence theorem for solutions of ordinary differential equations.

Therefore assume that $n>1$ and let $\tilde{A}_{1}$ be a matrix solution of

$$
\partial_{1} \tilde{A}_{1}=A_{1} \tilde{A}_{1},
$$


with initial condition $\tilde{A}_{1}\left(p_{0}\right)=I$. We then try to find a solution of the form $f=\tilde{A}_{1} g$, where $g={ }^{t}\left(g_{1}, \ldots, g_{m}\right)$ depends only on the variables $x_{2}, \ldots, x_{n}$. Clearly, we have that

$$
\partial_{1} f=A_{1} \tilde{A}_{1} g=A_{1} f .
$$

We also have that

$$
\begin{gathered}
\partial_{i} f=A_{i} f=A_{i} \tilde{A}_{1} g, \\
\partial_{i} f=\left(\partial_{i} \tilde{A}_{1}\right) g+\tilde{A}_{1} \partial_{i} g .
\end{gathered}
$$

As the matrix $\tilde{A}_{1}$ is locally invertible, it follows that the function $g$ has to satisfy

$$
\partial_{i} g=B_{i} g,
$$

where $B_{i}=-\tilde{A}_{1}^{-1}\left(\partial_{i} \tilde{A}_{1}\right)+\tilde{A}_{1}^{-1} A_{i} \tilde{A}_{1}$. Using that $\tilde{A}_{1}^{-1} \tilde{A}_{1}=I$ and therefore

$$
\partial_{i} \tilde{A}_{1}^{-1}=-\tilde{A}_{1}^{-1}\left(\partial_{i} \tilde{A}_{1}\right) \tilde{A}_{1}^{-1},
$$

we get that

$$
\begin{aligned}
\partial_{1} B_{i}= & \tilde{A}_{1}^{-1}\left(\partial_{1} \tilde{A}_{1}\right) \tilde{A}_{1}^{-1}\left(\partial_{i} \tilde{A}_{1}\right)-\tilde{A}_{1}^{-1}\left(\partial_{i} \partial_{1} \tilde{A}_{1}\right) \\
& -\tilde{A}_{1}^{-1}\left(\partial_{1} \tilde{A}_{1}\right) \tilde{A}_{1}^{-1} A_{i} \tilde{A}_{1}+\tilde{A}_{1}^{-1}\left(\partial_{1} A_{i}\right) \tilde{A}_{1}+\tilde{A}_{1}^{-1} A_{i}\left(\partial_{1} \tilde{A}_{1}\right) \\
= & \tilde{A}_{1}^{-1} \tilde{A}_{1}\left(\partial_{i} \tilde{A}_{1}\right)-\tilde{A}_{1}^{-1} \partial_{i}\left(A_{1} \tilde{A}_{1}\right) \\
& -\tilde{A}_{1}^{-1} A_{1} A_{i} \tilde{A}_{1}+\tilde{A}_{1}^{-1}\left(\partial_{1} A_{i}\right) \tilde{A}_{1}+\tilde{A}_{1}^{-1} A_{i} A_{1} \tilde{A}_{1} \\
= & -\tilde{A}_{1}^{-1}\left(\partial_{i} A_{1}-\partial_{1} A_{i}+A_{1} A_{i}-A_{i} A_{1}\right) \tilde{A}_{1}=0 .
\end{aligned}
$$

Similarly, we also obtain that

$$
\partial_{i} B_{j}-\partial_{j} B_{i}-\left[B_{i}, B_{j}\right]=\tilde{A}_{1}^{-1}\left(\partial_{i} A_{j}-\partial_{j} A_{i}-\left[A_{i}, A_{j}\right]\right) \tilde{A}_{1}=0 .
$$

Applying the induction hypothesis now completes the proof.

Lemma 10. Let $q \in M$. Then there exist vector fields $Z_{1}, \ldots, Z_{m}$ defined on a neighborhood of $q$, which form a basis of $T_{0}$ satisfying that

$$
\nabla_{X} Z_{k}=0
$$

for any vector field $X$.

Proof. We start with an arbitrary local basis $Z_{1}, \ldots, Z_{m}$. Any other basis $\tilde{Z}_{1}, \ldots, \tilde{Z}_{m}$ can then be written as $\tilde{Z}_{k}=\sum_{l=1}^{m} b_{k l} Z_{l}$. Since $T_{0}$ is parallel, we can also introduce 1-forms $\beta_{k}^{l}$ on $M$ by $\nabla_{X} Z_{k}=\sum_{l=1}^{m} \beta_{k}^{l}(X) Z_{l}$. Since $Z_{k} \in T_{0}$, it follows from the Gauss equation that

$$
\begin{aligned}
R(X, Y) Z_{k} & =A_{h\left(Y, Z_{k}\right)} X-A_{h\left(X, Z_{k}\right)} Y \\
& =(1 /(n-1)) \operatorname{Ric}(X, Y) Z_{k}-(1 /(n-1)) \operatorname{Ric}(Y, X) Z_{k} \\
& =0 .
\end{aligned}
$$


Therefore, we get that

$$
\begin{aligned}
0 & =R(X, Y) Z_{k} \\
& =\nabla_{X} \nabla_{Y} Z_{k}-\nabla_{Y} \nabla_{X} Z_{k}-\nabla_{[X, Y]} Z_{k} \\
& =\nabla_{X}\left(\sum_{l=1}^{m} \beta_{k}^{l}(Y) Z_{l}\right)-\nabla_{Y}\left(\sum_{l=1}^{m} \beta_{k}^{l}(X) Z_{l}\right)-\sum_{l=1}^{m} \beta_{k}^{l}([X, Y]) Z_{l} \\
& =\sum_{l=1}^{n} d \beta_{k}^{l}(X, Y) Z_{l}+\sum_{l, s=1}^{m}\left(\beta_{k}^{l}(Y) \beta_{l}^{s}(X)-\beta_{k}^{l}(X) \beta_{l}^{s}(Y)\right) Z_{s}
\end{aligned}
$$

from which it follows that

$$
d \beta_{k}^{l}(X, Y)+\sum_{s=1}^{m}\left(\beta_{k}^{s}(Y) \beta_{s}^{l}(X)-\beta_{k}^{s}(X) \beta_{s}^{l}(Y)\right)=0 .
$$

Now, expressing that the new basis $\tilde{Z}_{1}, \ldots, \tilde{Z}_{m}$ is parallel, we obtain that

$$
\begin{aligned}
0 & =\nabla_{X} \tilde{Z}_{k}=\sum_{l=1}^{m}\left(X\left(b_{k l}\right) Z_{l}+b_{k l} \nabla_{X} Z_{l}\right) \\
& =\sum_{l=1}^{m} X\left(b_{k l}\right) Z_{l}+\sum_{l, s=1}^{m} b_{k l} \beta_{l}^{s}(X) Z_{s} .
\end{aligned}
$$

Hence in order to obtain the desired basis, the functions $b_{k l}$ have to satisfy the following system of differential equations:

$$
X\left(b_{k l}\right)=-\sum_{s=1}^{m} \beta_{s}^{l}(X) b_{k s} .
$$

Notice that this is a system of differential equations as treated in Lemma 9. From Lemma 9, we get that a sufficient condition for the existence of a solution is that

$$
0=-d \beta_{\tilde{s}}^{l}(X, Y)+\sum_{s=1}^{m}\left(\beta_{s}^{l}(Y) \beta_{\tilde{s}}^{s}(X)-\beta_{s}^{l}(X) \beta_{\tilde{s}}^{s}(Y)\right),
$$

which is satisfied because of (25).

Let $q$ be a point of $M$ and assume that $X_{1}, \ldots, X_{r}$ form a local basis of a complementary distribution $T_{1}$ to $T_{0}$. Since the Ricci tensor restricted to the space $T_{1}$ is a nondegenerate symmetric bilinear form, we may assume that $X_{1}, \ldots, X_{r}$ form a pseudo-orthonormal basis, i.e., we may assume that

$$
\operatorname{Ric}\left(X_{i}, X_{j}\right)=((n-1) / 4) \varepsilon_{i} \delta_{i j}
$$

where $\varepsilon_{i}= \pm 1$. We also take a basis $Z_{1}, \ldots, Z_{m}$ of $T_{0}$ as constructed before. We introduce one forms $\omega_{i}^{j}$ and $v_{i}^{k}, 1 \leq i, j \leq r, 1 \leq k \leq m$, by

$$
\nabla_{X} X_{i}=\sum_{j=1}^{r} \omega_{i}^{j}(X) X_{j}+\sum_{k=1}^{m} v_{i}^{k}(X) Z_{k}
$$


Then, taking arbitrary vector fields $X$ and $Y$, it follows that

$$
\begin{aligned}
R(X, Y) X_{i}= & \nabla_{X} \nabla_{Y} X_{i}-\nabla_{Y} \nabla_{X} X_{i}-\nabla_{[X, Y]} X_{i} \\
= & \nabla_{X}\left(\sum_{j=1}^{r} \omega_{i}^{j}(Y) X_{j}+\sum_{k=1}^{m} v_{i}^{k}(Y) Z_{k}\right)-\nabla_{Y}\left(\sum_{j=1}^{r} \omega_{i}^{j}(X) X_{j}+\sum_{k=1}^{m} v_{i}^{k}(X) Z_{k}\right) \\
& -\sum_{j=1}^{r} \omega_{i}^{j}([X, Y]) X_{j}-\sum_{k=1}^{m} \nu_{i}^{k}([X, Y]) Z_{k} \\
= & \sum_{j=1}^{r} d \omega_{i}^{j}(X, Y) X_{j}+\sum_{k=1}^{m} d \nu_{i}^{k}(X, Y) Z_{k}+\sum_{j=1}^{r}\left(\omega_{i}^{j}(Y) \nabla_{X} X_{j}-\omega_{i}^{j}(X) \nabla_{Y} X_{j}\right) \\
= & \sum_{j=1}^{r} d \omega_{i}^{j}(X, Y) X_{j}+\sum_{j, j_{1}=1}^{r}\left(\omega_{i}^{j}(Y) \omega_{j}^{j_{1}}(X)-\omega_{i}^{j}(X) \omega_{j}^{j_{1}}(Y)\right) X_{j_{1}} \\
& +\sum_{k=1}^{m} d v_{i}^{k}(X, Y) Z_{k}+\sum_{j=1}^{r} \sum_{k=1}^{m}\left(\omega_{i}^{j}(Y) v_{j}^{k}(X)-\omega_{i}^{j}(X) v_{j}^{k}(Y)\right) Z_{k} \\
= & \sum_{j_{1}=1}^{r}\left(d \omega_{i}^{j_{1}}(X, Y)+\sum_{j=1}^{r}\left(\omega_{i}^{j}(Y) \omega_{j}^{j_{1}}(X)-\omega_{i}^{j}(X) \omega_{j}^{j_{1}}(Y)\right)\right) X_{j_{1}} \\
& +\sum_{k=1}^{m}\left(d \nu_{i}^{k}(X, Y)+\sum_{j=1}^{r}\left(\omega_{i}^{j}(Y) v_{j}^{k}(X)-\omega_{i}^{j}(X) v_{j}^{k}(Y)\right)\right) Z_{k} .
\end{aligned}
$$

On the other hand, using the Gauss equation, it follows that

$$
\begin{aligned}
R(X, Y) X_{i} & =A_{h\left(Y, X_{i}\right)} X-A_{h\left(X, X_{i}\right)} Y=0, \quad X, Y \in T_{0} \\
& =0, \quad Y \in T_{0}, X=X_{j}, j \neq i \\
& =-\varepsilon_{i} Y / 4, \quad Y \in T_{0}, X=X_{i} \\
& =0, \quad X=X_{j_{1}}, Y=X_{j_{2}}, \quad i, j_{1}, j_{2} \text { are mutually different } \\
& =-\varepsilon_{i} X_{j} / 4, \quad X=X_{i}, Y=X_{j}, i \neq j \\
& =0, \quad X=Y
\end{aligned}
$$

Next we will try to construct a more suitable local complementary distribution. It is clear that any other complementary distribution can be spanned by vector fields $\tilde{X}_{i}, 1 \leq i \leq r$, which are given by $\tilde{X}_{i}=X_{i}+\sum_{k=1}^{m} a_{i k} Z_{k}$, where the $a_{i k}$ are arbitrary local functions. The next lemma then shows how to define these functions to obtain a unique complementary distribution.

LEMMA 11. Let $q \in M$ and let $Z_{1}, \ldots, Z_{m}$ be a local basis of $T_{0}$ as constructed in Lemma 10. Then on a neighborhood $U$ of $q$ there exists a complementary distribution $T_{1}$ such 
that

$$
\begin{aligned}
& \nabla_{X} Y-(1 /(n-1)) \operatorname{Ric}(X, Y) Z^{*} \in T_{1}, \quad X, Y \in T_{1}, \\
& \nabla_{Z} X \in T_{1}, \quad X \in T_{1}, Z \in T_{0},
\end{aligned}
$$

where $Z^{*}=\sum_{k=1}^{m} g_{k} Z_{k} \in T_{0}$ is a vector field satisfying

$$
X\left(g_{k}\right)=0, \quad X \in T_{1} ; \quad Z_{l}\left(g_{k}\right)=\delta_{k l} .
$$

PROOF. We introduce a vector field $Z^{*}=\sum_{k=1}^{m} g_{k} Z_{k}$. We get that

$$
\begin{aligned}
\nabla_{Z_{k}} \tilde{X}_{i} & =\nabla_{Z_{k}} X_{i}+\sum_{l=1}^{m} Z_{k}\left(a_{i l}\right) Z_{l} \\
& =\sum_{j=1}^{r} \omega_{i}^{j}\left(Z_{k}\right) X_{j}+\sum_{l=1}^{m} v_{i}^{l}\left(Z_{k}\right) Z_{l}+\sum_{l=1}^{m} Z_{k}\left(a_{i l}\right) Z_{l} \\
& =\sum_{j=1}^{r} \omega_{i}^{j}\left(Z_{k}\right) \tilde{X}_{j}-\sum_{j=1}^{r} \sum_{l=1}^{m} \omega_{i}^{j}\left(Z_{k}\right) a_{j l} Z_{l}+\sum_{l=1}^{m} v_{i}^{l}\left(Z_{k}\right) Z_{l}+\sum_{l=1}^{m} Z_{k}\left(a_{i l}\right) Z_{l} \\
\nabla_{X_{j_{1}}} \tilde{X}_{i} & =\nabla_{X_{j_{1}}} X_{i}+\sum_{l=1}^{m} X_{j_{1}}\left(a_{i l}\right) Z_{l} \\
& =\sum_{j=1}^{r} \omega_{i}^{j}\left(X_{j_{1}}\right) X_{j}+\sum_{l=1}^{m} v_{i}^{l}\left(X_{j_{1}}\right) Z_{l}+\sum_{l=1}^{m} X_{j_{1}}\left(a_{i l}\right) Z_{l} \\
& =\sum_{j=1}^{r} \omega_{i}^{j}\left(X_{j_{1}}\right) \tilde{X}_{j}-\sum_{j=1}^{r} \sum_{l=1}^{m} \omega_{i}^{j}\left(X_{j_{1}}\right) a_{j l} Z_{l}+\sum_{l=1}^{m} v_{i}^{l}\left(X_{j_{1}}\right) Z_{l}+\sum_{l=1}^{m} X_{j_{1}}\left(a_{i l}\right) Z_{l}
\end{aligned}
$$

Therefore we see that the connection $\nabla$ has the form as described in the lemma if and only if the functions $a_{i k}$ satisfy the following system of differential equations:

$$
\begin{aligned}
& Z_{k}\left(a_{i l}\right)=-v_{i}^{l}\left(Z_{k}\right)+\sum_{j=1}^{r} \omega_{i}^{j}\left(Z_{k}\right) a_{j l} \\
& X_{j_{1}}\left(a_{i l}\right)=-v_{i}^{l}\left(X_{j_{1}}\right)+\sum_{j=1}^{r} \omega_{i}^{j}\left(X_{j_{1}}\right) a_{j l}+(1 / 4) \varepsilon_{i} \delta_{j_{1}} g_{l} .
\end{aligned}
$$

The second part of the lemma now reduces to the following system of differential equations:

$$
X_{j}\left(g_{l}\right)=-a_{j l}, \quad Z_{k}\left(g_{l}\right)=\delta_{k l} .
$$

Therefore, in order to complete the proof of the lemma, we notice that this is again a system of differential equations as treated in Lemma 9 (with functions $a_{i l}$ and $g_{l}$ ). Using the notation of Lemma 9, we see that it is sufficient to show that the following system of differential 
equations:

$$
X\left(f_{i}\right)=\sum_{j=0}^{n} \alpha_{i j}(X) f_{j}+\beta_{i}(X), \quad i=0,1, \ldots, n
$$

has a solution, where for some fixed $l$ and $i, j=1, \ldots, r$, we have that

$$
\begin{array}{ll}
f_{0}=g_{l}, & \\
f_{i}=a_{i l}, & \\
\alpha_{00}=0, & \alpha_{0 i}\left(Z_{k}\right)=0, \\
\alpha_{0 i}\left(X_{j}\right)=-\delta_{i j}, & \alpha_{i 0}\left(Z_{k}\right)=0, \\
\alpha_{i 0}\left(X_{j}\right)=(1 / 4) \varepsilon_{i} \delta_{i j}, & \\
\alpha_{i j}(X)=\omega_{i}^{j}(X), & \beta_{0}\left(Z_{k}\right)=\delta_{k l}, \\
\beta_{0}\left(X_{j}\right)=0, & \\
\beta_{i}(X)=-v_{i}^{l}(X) . &
\end{array}
$$

Using the above definitions and (28), we see that for $i, j>0$, we have that:

$$
\begin{aligned}
& d \alpha_{i j}(X, Y)-\sum_{j_{2}=0}^{r}\left(\alpha_{i j_{2}}(X) \alpha_{j_{2} j}(Y)-\alpha_{i j_{2}}(Y) \alpha_{j_{2} j}(X)\right) \\
& =d \omega_{i}^{j}(X, Y)-\sum_{j_{2}=1}^{r}\left(\omega_{i}^{j_{2}}(X) \omega_{j_{2}}^{j}(Y)-\omega_{i}^{j_{2}}(Y) \omega_{j_{2}}^{j}(X)\right) \\
& -\alpha_{i 0}(X) \alpha_{0 j}(Y)+\alpha_{i 0}(Y) \alpha_{0 i}(X) \\
& =\left(R(X, Y) X_{i}\right)^{X_{j} \text {-component }}+\alpha_{i 0}(X) Y^{X_{j} \text {-component }}-\alpha_{i 0}(Y) X^{X_{j} \text {-component. }}
\end{aligned}
$$

Using now (29) up to (33) it follows that the righthandside vanishes identically and therefore

$$
d \alpha_{i j}(X, Y)-\sum_{j_{2}=0}^{r}\left(\alpha_{i j_{2}}(X) \alpha_{j_{2} j}(Y)-\alpha_{i j_{2}}(Y) \alpha_{j_{2} j}(X)\right)=0
$$

The other equations can be verified in a similar way.

Next, we introduce a metric $g$ on $U$ by

$$
\begin{aligned}
& g(X, Y)=(4 /(n-1)) \operatorname{Ric}(X, Y), \quad X, Y \in T_{1}, \\
& g(X, Z)=0, \quad X \in T_{1}, Z \in T_{0}, \\
& g\left(Z_{k}, Z_{l}\right)=\delta_{k l}, \quad 1 \leq k, l \leq m .
\end{aligned}
$$


LEMMA 12. The Levi-Civita connection $\hat{\nabla}$ of the metric $g$ is given by

$$
\begin{aligned}
& \hat{\nabla}_{X} Y=\nabla_{X} Y-(1 / 4) g(X, Y) Z^{*}, \quad X, Y \in T_{1}, \\
& \hat{\nabla}_{Z} X=\nabla_{Z} X, \quad X \in T_{1}, Z \in T_{0}, \\
& \hat{\nabla}_{X} Z_{k}=0, \quad X \in T_{1}, 1 \leq k \leq m, \\
& \hat{\nabla}_{Z_{k}} Z_{l}=0, \quad 1 \leq k, l \leq m .
\end{aligned}
$$

Proof. We define a connection by the above formulas. Since $\nabla$ is symmetric, it follows from the definition that $\hat{\nabla}$ is symmetric too. So we have only to check that $\hat{\nabla}$ is compatible with the metric $g$. Let $X$ be an arbitrary tangent vector field. First we take $Y_{1}, Y_{2} \in T_{1}$. Then, since Ric is parallel with respect to $\nabla$, it follows that

$$
\begin{aligned}
\left(\hat{\nabla}_{X} g\right)\left(Y_{1}, Y_{2}\right) & =X g\left(Y_{1}, Y_{2}\right)-g\left(\hat{\nabla}_{X} Y_{1}, Y_{2}\right)-g\left(Y_{1}, \hat{\nabla}_{X} Y_{2}\right) \\
& =(4 /(n-1)) X \operatorname{Ric}\left(Y_{1}, Y_{2}\right)-g\left(\nabla_{X} Y_{1}, Y_{2}\right)-g\left(Y_{1}, \nabla_{X} Y_{2}\right) \\
& =(4 /(n-1))\left(X \operatorname{Ric}\left(Y_{1}, Y_{2}\right)-\operatorname{Ric}\left(\nabla_{X} Y_{1}, Y_{2}\right)-\operatorname{Ric}\left(Y_{1}, \nabla_{X} Y_{2}\right)\right) \\
& =0 .
\end{aligned}
$$

Next we compute for $Y \in T_{1}$ and $Z \in T_{0}$ that

$$
\begin{aligned}
\left(\hat{\nabla}_{X} g\right)(Y, Z) & =X g(Y, Z)-g\left(\hat{\nabla}_{X} Y, Z\right)-g\left(Y, \hat{\nabla}_{X} Z\right) \\
& =-g\left(\hat{\nabla}_{X} Y, Z\right)=0
\end{aligned}
$$

since by construction $\hat{\nabla}_{X} Y$ does not have a $T_{0}$ component. The fact that $\left(\hat{\nabla}_{X} g\right)\left(Z_{k}, Z_{l}\right)=0$ follows trivially.

As a consequence, we have

THEOREM 1. Let $\phi: M \rightarrow \boldsymbol{R}^{n+n(n+1) / 2}$ be a parallel linearly full affine immersion and let $q \in M$. Then there exists a metric $g$ on a neighborhood $V$ of $q$ in $M$ such that $V$ is isometric to an open set of $S_{s}^{r}(2) \times \boldsymbol{R}^{m}$. Moreover, the affine connection $\nabla$ is related to the Levi-Civita connection of $S_{S}^{r}(2) \times \boldsymbol{R}^{m}$ by

$$
\begin{aligned}
& \nabla_{X} Y=\hat{\nabla}_{X} Y+(1 / 4) g(X, Y) \sum_{k=1}^{m} z_{k} \partial_{k}, \\
& \nabla_{X} \partial_{k}=0, \quad \nabla_{\partial k} X=0, \quad \nabla_{\partial k} \partial l=0,
\end{aligned}
$$

where $X, Y$ are tangent to $S_{S}^{r}(2)$ (and we consider them as tangent vectors of the product manifold in the standard way) and $\partial_{k}, k=1, \ldots, m$, is the standard basis of $\boldsymbol{R}^{m}$ with coordinates denoted by $z_{1}, \ldots, z_{k}$.

Proof. Let $q \in M$. It follows from the previous lemmas that both distributions $T_{0}$ and $T_{1}$ are parallel with respect to $\hat{\nabla}$. Consequently, applying the results of [15] and [16], we get that a neighborhood $V$ of the point $q$ is congruent with the semi-Riemannian product manifold $M_{1} \times M_{0}$. Here, we interprete $T_{1}$ as tangent to $M_{1}$ and $T_{0}$ as tangent to $M_{0}$. 
From the definition of $\hat{\nabla}$ it follows that $Z_{1}, \ldots, Z_{m}$ are parallel vector fields of unit length on $M_{0}$, implying that $M_{0}$ is congruent with $\boldsymbol{R}^{m}$ and that we can interprete the vector fields $Z_{k}$ as coordinate vector fields $Z_{k}=\partial_{k}=\partial / \partial z_{k}$. Writing now the vector field $Z^{*}=$ $\sum_{k=1}^{m} g_{k} Z_{k}$ and recalling that the functions $g_{k}$ depended only on $M_{0}$ and were determined there by the following system of differential equations:

$$
\partial_{l} g_{k}=\delta_{k l},
$$

we get that, after applying a translation of the coordinates, if necessary, that $g_{k}=z_{k}$.

Now, we still have to show that $M_{1}$ is locally isometric to $S_{s}^{r}(2)$. Since all one-dimensional manifolds are locally isometric, we may assume that $r \geq 2$. In that case, we have for $Y_{1}$ and $Y_{2}$ tangent to $M_{1}$ that

$$
\begin{aligned}
g\left(\hat{R}\left(Y_{1}, Y_{2}\right) Y_{2}, Y_{1}\right)= & g\left(\hat{\nabla}_{Y_{1}} \hat{\nabla}_{Y_{2}} Y_{2}-\hat{\nabla}_{Y_{2}} \hat{\nabla}_{Y_{1}} Y_{2}-\hat{\nabla}_{\left[Y_{1}, Y_{2}\right]} Y_{2}, Y_{1}\right) \\
= & g\left(\nabla_{Y_{1}}\left(\nabla_{Y_{2}} Y_{2}-(1 / 4) g\left(Y_{2}, Y_{2}\right) Z^{*}\right)-\nabla_{Y_{2}}\left(\nabla_{Y_{1}} Y_{2}-(1 / 4) g\left(Y_{1}, Y_{2}\right) Z^{*}\right)\right. \\
& \left.-\nabla_{\left[Y_{1}, Y_{2}\right]} Y_{2}, Y_{1}\right) \\
= & g\left(R\left(Y_{1}, Y_{2}\right) Y_{2}, Y_{1}\right) \\
= & g\left(A_{h\left(Y_{2}, Y_{2}\right)} Y_{1}, Y_{1}\right)-g\left(A_{h\left(Y_{1}, Y_{2}\right)} Y_{2}, Y_{1}\right) \\
= & (1 / 2) g\left(Y_{1}, Y_{2}\right)^{2}+(1 / 2) g\left(Y_{2}, Y_{2}\right) g\left(Y_{1}, Y_{1}\right)-(3 / 4) g\left(Y_{1}, Y_{2}\right)^{2} \\
& -(1 / 4) g\left(Y_{2}, Y_{2}\right) g\left(Y_{1}, Y_{1}\right) \\
= & (1 / 4)\left(g\left(Y_{2}, Y_{2}\right) g\left(Y_{1}, Y_{1}\right)-g\left(Y_{1}, Y_{2}\right)^{2}\right) .
\end{aligned}
$$

Consequently, $M_{1}$ has constant sectional curvature $1 / 4$ and is therefore congruent with the semi-Riemannian sphere of radius 2 and index $s$.

The remaining formulas now follow from the previous lemmas together with the fact that $X$ and $Y$ are tangent to $S_{s}^{r}(2)$.

We are now in a position to prove the main classification result of the paper.

THEOREM 2. Let $\phi: M \rightarrow \boldsymbol{R}^{n+n(n+1) / 2}$ be a parallel linearly full affine immersion. Then $M$ is affine congruent with one of the affine immersions described in Section 2.

Proof. Let $q \in M$. By Theorem 1, we have that a neighborhood of $q$ is isometric to an open set of $S_{s}^{r}(2) \times \boldsymbol{R}^{m}$. We consider the corresponding immersion $F$. We indicate the immersion under consideration by adding an appropriate superscript. By construction both $F$ and $\phi$ have the same induced affine connection. It is also clear that by an affine transformation we may assume that $F(q)=\phi(q), F_{*}(v)=\phi_{*}(v)$ and $h^{F}(v, w)=h^{\phi}(v, w)$, where $v$ and $w$ are tangent vectors at the point $q$.

Let $\gamma$ be a geodesic, with respect to $\nabla$, in $V$. We denote by $\gamma_{1}=\phi(\gamma)$ and by $\gamma_{2}=F(\gamma)$. Then, using the previous lemmas we have that 


$$
\begin{aligned}
& \gamma_{1}^{\prime}=\phi_{*}\left(\gamma^{\prime}\right), \\
& \gamma_{1}^{\prime \prime}=h^{\phi}\left(\gamma^{\prime}, \gamma^{\prime}\right), \\
& \gamma_{1}^{\prime \prime \prime}=-\phi_{*}\left(A_{h^{\phi}\left(\gamma^{\prime}, \gamma^{\prime}\right)} \gamma^{\prime}\right)=-(4 /(n-1)) \operatorname{Ric}\left(\gamma^{\prime}, \gamma^{\prime}\right) \gamma_{1}^{\prime} .
\end{aligned}
$$

Similarly, we obtain that $\gamma_{2}^{\prime \prime \prime}=-(4 /(n-1)) \operatorname{Ric}\left(\gamma^{\prime}, \gamma^{\prime}\right) \gamma_{2}^{\prime}$. By the initial conditions and the uniqueness of solutions it follows that $F$ and $\phi$ coincide.

\section{REFERENCES}

[ 1 ] C. Blomstrom, Planar geodesic immersions in pseudo-Euclidean space, Math. Ann. 274(1986), 585-598.

[ 2 ] J. DePrEZ, Semi-parallel surfaces in Euclidean space, J. Geom. 25(1985), 192-200.

[ 3 ] F. Dillen and L. VRancken, Parallel hypersurfaces of affine spaces, Rend. Sem. Mat. Messina Ser. II 2(16)(1993), 71-80

[ 4 ] F. DiLlEN, Semi-parallel hypersurfaces of a real space form, Israel J. Math. 75(1991), 193-202.

[ 5 ] D. FERUS, Immersions with parallel second fundamental form, Math. Z. 140(1974), 87-93.

[6] M. MAGid AND L. VRANCKEN, Affine surfaces in $\boldsymbol{R}^{5}$ with zero cubic form, Differential Geom. Appli. 14(2001), 125-136.

[ 7 ] F. MERCURI, Parallel and semi-parallel immersions into space forms, Riv. Mat. Univ. Parma 17(1991), 91108.

[ 8 ] K. Nomizu And L. VRancken, A new equiaffine theory for surfaces in $\boldsymbol{R}^{4}$, Internat. J. Math. 4(1993), $127-165$.

[ 9 ] K. Nomizu And U. Pinkall, Cubic form theorem for affine immersions, Result. Math. 13(1988), 338-362.

[10] K. Nomizu and U. Pinkall, On the geometry of affine immersions, Math. Z. 195(1987), 165-178.

[11] B. O’NEILL, Isotropic and Kaehler immersions, Can. J. Math. 17(1965), 907-915.

[12] B. O’NeILl, Semi-Riemannian geometry with applications to relativity, Pure Appl. Math. 103, Academic Press Inc., New-York-London, 1983.

[13] K. H. WeISE, Der Beruehrungstensor zweier Flaechen und die Affingeometrie der $F_{p}$ im $A_{n}$. I, Math. Z. 43(1937), 469-480.

[14] K. H. WeISE, Der Beruehrungstensor zweier Flaechen und die Affingeometrie der $F_{p}$ im $A_{n}$. II, Math. Z. 44(1938), 161-184.

[15] H. Wu, Decomposition of Riemannian manifolds, Bull. Amer. Math. Soc. 70(1964), 610-617.

[16] H. WU, On the de Rham decomposition theorem, Illinois J. Math. 8(1964), 291-311.

MATHEMATISCH INSTITUUT

UNIVERSITEIT UTRECHT

BUDAPESTLAAN 6

3584 CD UTRECHT

THE NETHERLANDS

E-mail address: vrancken@math.uu.nl 